\title{
A multiscale framework for the prediction of concrete self-desiccation
}

\author{
M. Pathirage \\ Department of Civil and Environmental Engineering, McCormick School of Engineering and Applied Science, \\ Northwestern University, Evanston, IL, USA
}

D.P. Bentz

Engineering Laboratory, National Institute of Standards and Technology, Gaithersburg, MD, USA

G. Di Luzio

Department of Civil and Environmental Engineering, Politecnico di Milano, Milan, Italy

E. Masoero

School of Civil Engineering and Geosciences, Newcastle University, Newcastle upon Tyne, UK

\section{G. Cusatis}

Department of Civil and Environmental Engineering, McCormick School of Engineering and Applied Science, Northwestern University, Evanston, IL, USA

\begin{abstract}
Cement hydration in concrete and mortar has been studied thoroughly over the past 50 years. To fully understand hydration in concrete and predict the evolution of the hygral, thermal, and mechanical properties at the structural level, one needs to studynot only the reaction kinetics but also the development of the microstructure. Many models have been developed for this purpose, some of them looking only at the micro-scale or at the macro-scale and others tackling the fundamental nature of the issue, which can be qualified as a multiscale problem. This paper proposes a novel approach that consists of combining a cement hydration model at the microstructural level, the CEMHYD3D model, with a macroscopic hygro-thermo-chemical model, the HTC model. The coupling is performed by postprocessing the output of the CEMHYD3D model, in particular with reference to cement hydration degree, silica fume reaction degree, and amounts of evaporable water and chemically bound water in order to identify through a curve fitting routine the parameters of the HTC formulation. This approach allows the possibility of predicting concrete behavior at multiple scales based on the actual chemical and microstructural evolution, thus enhancing the capabilities of the so-called HTC-CEMHYD3D model. This paper focuses on 1) introducing the concepts behind the formulation of self-desiccation and 2) demonstrating the predictive capabilities of the coupled model using some available experimental data.
\end{abstract}

\section{INTRODUCTION}

The behavior of concrete in terms of its mechanical, hygral, and thermal properties has always been of a great interest to researchers and engineers, and many computational models have been proposed.

Models at the micro-scale level are numerous. The first model that described the hydration kinetics and development is the Jennings and Johnson microstructure simulation model (Jennings \& Johnson 1986). Cement particles are represented as spheres, and hydration is simulated through a concentric growth of Calcium-Silicate-Hydrate (CSH) layers surrounding unhydrated particles. The model is able to capture key hydration features, including particle size distribution and evolution of volume due the formation of reaction products and the consumption of reactants. However, several model limitations must be cited. For example, the model is only capable of simulating hydration of tricalcium silicate (C3S), and does not take into account transport phenomena.

The HymoStruc model (Van Breugel 1995, Koenders \& Van Breugel 1997, Ye, Van Breugel, \& Fraaij 2003) appeared as a continuation of this effort. It uses the same approach as in Jennings and Johnson's model, and statistical analysis is introduced. However, the previous limitations on transport phenomena, as well as the actual chemistry in the pore solution are still constraints to an extensive use of this model. 
The CEMHYD3D model (Bentz 1997, Bentz \& Garboczi 1990, Garboczi \& Bentz 1992, Bentz 2005) proposed a new approach that is based on cellular automata algorithm. CEMHYD3D stands for Cement Hydration in three dimensions and was developed at the National Institute of Standards and Technology (NIST). This open-source code is a digital image-based model and uses fundamental knowledge about hydration reactions and products. The model starts with an initial 3D microstructure, reconstructed from information on the actual cement powder via a scanning electron microscope (SEM) image for the phase distribution and the measured particle size distribution. Hydrating cement paste is represented by a cube of voxels. Each voxel is associated with a phase and can move and transform into another phase based on an iterative process through cycles. A database of cement types is available such that one needs only to calibrate a single parameter controlling the relationship between cycle lengths and real time. This model was successfully used in the literature and captures all aspects of the microstructure development, from chemical reactions to transport phenomena. It was legitimately pointed out (Thomas et al. 2011) that the parameter mapping the cycle lengths to the real time does not have a physical meaning and that the particle size limit of 1 voxel $\left(1 \mu \mathrm{m}^{3}\right)$ in the CEMHYD3D model brings a limitation. The HydratiCA model (Bullard 2007a, Bullard 2007b) and $\mu$ ic model (Navi \& Pignat 1996, Bishnoi \& Scrivener 2009) were developed in response to the aforementioned constraints. However, it is discussed later in this paper that such limitations have little or no effect on the proposed multiscale approach developed here. At the macroscale, several models can be found in the literature that simulate not only cement hydration but also hygro-thermo-mechanical properties of concrete. Among others, the models of Ulm \& Coussy (1995) and De Schutter \& Taerwe (1996) are able to describe strength increase due to cement hydration. The temperature effect on hydration is taken into account in the work of Cervera, Oliver, \& Prato (1999). More complex models provide better predictive capabilities in terms of hydration process, flow phenomena,chemical shrinkage and selfdesiccation (Gawin, Pesavento, \& Schrefler 2006a, Gawin, Pesavento, \& Schrefler 2006b, Pan et al. 2017, Rahimi-Aghdam, Bažant, \& Qomi 2017). Di Luzio \& Cusatis (2009) proposed a hygrothermo-chemical model, called the HTC model, that takes into account moisture transport governing the porerelative humidity and heat transfer. It also accounts for the heat released by hydration, silica fume reaction and cement hydration degrees, adsorption/desorption isotherms (by estimating the chemically bound water and the evaporable water), and concrete permeability. This model is considered in this paper.

It must be noted that in most of these models, the use of empirical or semi-empirical formulations at the macro-scale do not allow a fundamental description of physics and chemistry at a lower scale. Moreover, for almost all the models, lengthy and sometimes complex calibration processes are needed, which limit the use of such models to cases in which available and up-to-date experimental data exist.

The present study introduces a multiscale approach, coupling the CEMHYD3D model to the HTC model, that overcomes the aforementioned limitations.

\section{THE HTC-CEMHYD3D MODEL}

\subsection{Overview of the CEMHYD3D model}

The model considers the cement particles as digitized spherical elements. These spheres are created to replicate the measured particle size distribution and phase distribution obtained from SEM analysis on a generic volume of one million cubic micrometers. Each of the spheres are made of adjacent one micrometer cube voxels that represent either cement clinker phases, calcium sulfates, or supplementary cementitious materials. All the particles lie in water, also represented with voxels; the microstructureis then computed to form a representative volume element. The hydration process is computed through a cellular automata algorithm, where cement phase voxels dissolve, then diffuse where capillary water exists, and collide with other reactants to produce solid hydration products. This process occurs in a series of cycles that cumulatively represent the termination time of the simulation. The volume stoichiometry is conserved for the hydration reactions in the unit volume, and periodic boundaries are used to ensure an overall homogeneous distribution of reactants and hydration products. To control the kinetics of the chemical reactions, a real time is mapped to the number of hydration cycles through a parameter that usually needs to be calibrated from experimental data on hydration degree or non-evaporable water content over time. In order to make the model parameter-free, a commonly used conversion factor of 0.00035 hours $/$ cycle $^{2}$ (Bentz 2005) is taken and fixed for all the simulations shown later inthe study.

\subsection{Self-desiccation formulation in the HTC model}

The HTC model is a macro-scale model. It considers the moisture transport and heat transfer through coupled diffusion equations (Di Luzio \& Cusatis 2009). The present work focuses only on 
self-desiccation, which involves the adsorption/ desorption isotherms, thecement hydration degree $\alpha_{c}$, evaporable water $w_{e}$, and non-evaporable water $w_{n}$. Silica fume reaction degree $\alpha_{s}$ is also taken into account. Since the gradient of temperature and humidity in space are minimal or zero regarding the size of the samples used to study selfdesiccation, one can formulate the following moisture mass balance equation at any time $t$ as

$$
\frac{\partial w_{e}}{\partial h} \frac{\partial h}{\partial t}+\frac{\partial w_{e}}{\partial \alpha_{c}} \dot{\alpha}_{c}+\frac{\partial w_{e}}{\partial \alpha_{s}} \dot{\alpha}_{s}+\dot{w}_{n}=0
$$

where $h$ is the internal moisture or relative humidity. The cement hydration degree is formulated as

$$
\begin{aligned}
& \dot{\alpha}_{c}=A_{c 1}\left(A_{c 2}+\alpha_{c}\right)\left\langle\alpha_{c}^{\infty}-\alpha_{c}\right\rangle e^{-\frac{\eta_{c} \alpha_{c}}{\alpha_{c}^{\omega}}} e^{-\frac{t_{a c}}{R T}} \\
& \alpha_{c}^{\infty}(h)=\alpha_{c}^{\infty, s a t} \gamma(h) \\
& \gamma(h)=e^{h_{c}^{0}\left(1-\frac{1}{h}\right)}
\end{aligned}
$$

where $A_{c 1}, A_{c 2}=0.005, \eta_{c}$ and $h_{c}^{0}$ are material parameters, $\alpha_{c}^{\infty}$ is the asymptotic hydration degree, $\alpha_{s}^{\infty, s a t}$ is the asymptotic hydration degree at complete saturation, $E_{a c}$ is the hydration activation energy, $R$ is the universal gas constant. One can notice that in equation 2 , the term $\alpha_{c}^{\infty}-\alpha_{c}$ can be negative when $h$ is small enough, which would lead to an unphysical reduction in hydration degree over time. To ensure a positive rate of cement hydration degree, the Macaulay brackets $\langle\rangle=.\max (0,$.$) are used.$

The silica fume reaction degree is written in a similar functional form as

$$
\begin{aligned}
& \dot{\alpha}_{s}=A_{s 1}\left(A_{s 2}+\alpha_{s}\right)\left\langle\alpha_{s}^{\infty}-\alpha_{s}\right\rangle e^{-\frac{\eta_{1} \alpha_{s}}{\alpha_{s}^{\infty}}} e^{-\frac{E_{a s}}{R T}} \\
& \alpha_{s}^{\infty}(h)=\alpha_{s}^{\infty, s a t} \gamma(h) \\
& \gamma(h)=e^{h_{s}^{0}\left(1-\frac{1}{h}\right)}
\end{aligned}
$$

where $A_{s 1}, A_{s 2}=0.05, \eta_{s}$ and $h_{s}^{0}$ are material parameters, $\alpha_{s}^{\infty}$ is the asymptotic hydration degree, $\alpha_{s}^{\infty, s a t}$ is the asymptotic hydration degree at saturation, and $E_{a s}$ is the silica fume reaction activation energy. The notation $\langle$.$\rangle is once again used in equation 5$ to avoid an unphysical decrease of silica fume reaction degree over time.

The cement hydration degree and the silica fume reaction degree are taken into account in a single formulation, namely the total reaction degree (Di Luzio \& Cusatis 2013) as

$$
\alpha=\frac{\alpha_{c} c \tilde{Q}_{c}^{\infty}+\alpha_{s} s \tilde{Q}_{s}^{\infty}}{c \tilde{Q}_{c}^{\infty} \alpha_{c}^{\infty}+s \tilde{Q}_{s}^{\infty} \alpha_{s}^{\infty}}
$$

where $\tilde{Q}_{c}^{\infty}$ and $\tilde{Q}^{\infty}$ are the latent heat of hydration reaction and silica fume reaction, respectively, per unit of hydrated cementitious material, $c$ and $s$ are respectively the cement and silica fume contents. The chemically bound water is commonly formulated as $w_{n}=\kappa_{c} \alpha_{c} c$, where $\kappa_{c}$ is the mass ratio of non-evaporable water at full hydration and $c$ is the cement content. Furthermore, one can define $w_{e}$, the evaporable water, which is the resultant of two contributions, i.e., the evaporable water present in the CSH gel $w_{e}^{\text {gel }}$ and the capillary water $w_{e}^{\text {cap }}$ : $w_{e}=w_{e}^{\text {gel }}+w_{e}^{\text {cap }}$. They are formulated as

$$
w_{e}^{g e l}=g_{2} \alpha\left(c \alpha_{c}^{\infty}+s \alpha_{s}^{\infty} \frac{\tilde{Q}_{s}^{\infty}}{\tilde{Q}_{c}^{\infty}}\right)\left[1-e^{-10 \alpha_{c}^{\infty}\left(g_{1}-\alpha\right)^{h}}\right]
$$

where $g_{1}=1.5$ and $g_{2}$ are material parameters. The capillary water is computed through a mass balance

$w_{0}+\Delta w=w_{e}^{g e l}+w_{e}^{c a p}+w_{n}$

where $w_{0}$ is the initial water content. $\Delta w$ is defined as the mass of water to be supplied to compensate chemical shrinkage and maintain saturated conditions. It can be written as

$$
\Delta w=X_{c}\left[\left(1-\frac{\tilde{Q}_{c}^{\infty}}{\tilde{Q}_{s}^{\infty}}\right) c \alpha_{c}+s \alpha_{s}\right]
$$

where $X_{c}$ is a material parameter. The equation 1 constitutes the governing equation and is discretized and solved using an implicit Crank-Nicolson method.

\subsection{Multiscale identification of the HTC model parameters}

Relevant outputs of the CEMHYD3D model are post-processed, and the material parameters of the HTC model listed earlier are automatically fitted. The identification procedure is performed under saturated and sealed conditions.

At saturation, i.e., $h=1$, the parameters $A_{c 1}, \eta_{c}$, $\alpha_{c}^{\infty, s a t}$ in Equations 2 and 3 and $A_{s 1}, \eta_{s}, \alpha_{s}^{\infty, \text { sat }}$ in Equations 5 and 6 are identified from the curves for cement hydration and the silica fume reaction degrees versus time obtained by the CEMHYD3D model. The activation energies for silica fume and cement reactions are the ones used in the microscale model. $\tilde{Q}_{c}^{\infty}$ and $\tilde{Q}_{s}^{\infty}$ in Equation 8 are identified by computing the initial masses of the different clinker phases and silica fume. In addition, one can compute the non-evaporable water by assuming that, for each cement clinker phase, the mass of chemically bound water produced is 
proportional to the mass of reacted phase, through constant coefficients (Molina 1992). This allows the identification of $\kappa_{c}$. The evaporable water in the gel is computed from the CEMHYD3D model, along with the chemical shrinkage, allowing the identification of the parameters $g_{2}$ and $X_{c}$. The capillary water content is then evaluated from Equation 10, knowing $w_{0}$.

Last, the CEMHYD3D model evaluates the cement hydration and silica fume reaction degrees curves in time for the sealed condition. This enables the identification of the two remaining parameters $h_{c}^{0}$ and $h_{s}^{0}$ in Equations 4 and 7.

\section{PREDICTION RESULTS}

The HTC-CEMHYD3D model was used to predict experimental data on self-desiccation (Persson 1996, Persson 1997, Persson 1998). Three different concrete mixes were used, with a wide range of water to cement ratios, i.e., $w / c=0.22, w / c=$ 0.36 , and $w / c=0.48$ and a constant silica fume to cement mass ratio of $s / c=0.1$. Concrete specimens were sealed and the relative humidity was measured at 28, 90, and 450 days after casting. For the mix design, a low alkali cement was used, which was replicated by using a similar cement type in the HTC-CEMHYD3D model. Table 1 shows the material parameters that were identified using the CEMHYD3D model and transferred to the HTC model.

Results are shown in Figure 1. The predictions are in agreement with the experimental data and able to capture the decrease in humidity at various

Table 1. Identified HTC model parameters from CEMHYD3D.

\begin{tabular}{lllll}
\hline & & $w / c=0.22$ & $w / c=0.36$ & $\begin{array}{l}w / c=0.48 \\
\text { HTC }\end{array}$ \\
\hline$A_{c 1}$ & {$[1 / \mathrm{h}]$} & $9.67 \times 10^{6}$ & $5.76 \times 10^{6}$ & $2.78 \times 10^{6}$ \\
$\eta_{c}$ & {$[-]$} & 4.42 & 5.49 & 6.62 \\
$\alpha_{c}^{\infty, s a t}$ & {$[-]$} & 0.38 & 0.55 & 0.83 \\
$E_{a c}$ & {$[\mathrm{~kJ} / \mathrm{mole}]$} & 40.00 & 40.00 & 40.00 \\
$h_{c}^{0}$ & {$[-]$} & 0.20 & 0.40 & 0.50 \\
$A_{s 1}$ & {$[1 / \mathrm{h}]$} & $1.17 \times 10^{14}$ & $9.97 \times 10^{13}$ & $7.71 \times 10^{13}$ \\
$\eta_{s}$ & {$[-]$} & 0.84 & 2.08 & 1.86 \\
$\alpha_{s}^{\omega, s a t}$ & {$[-]$} & 0.49 & 0.76 & 0.84 \\
$E_{a s}$ & {$[\mathrm{~kJ} / \mathrm{mole}]$} & 83.14 & 83.14 & 83.14 \\
$h_{s}^{0}$ & {$[-]$} & 1.50 & 0.55 & 0.40 \\
$\tilde{Q}_{c}^{\infty}$ & {$[\mathrm{kJ} / \mathrm{kg}]$} & 506.74 & 508.04 & 506.52 \\
$\tilde{Q}_{s}^{\infty}$ & {$[\mathrm{kJ} / \mathrm{kg}]$} & 780.00 & 780.00 & 780.00 \\
$\kappa_{c}$ & {$[-]$} & 0.27 & 0.26 & 0.25 \\
$g_{2}$ & {$[-]$} & 0.32 & 0.29 & 0.24 \\
$X_{c}$ & {$[-]$} & 0.22 & 0.21 & 0.23 \\
\hline
\end{tabular}
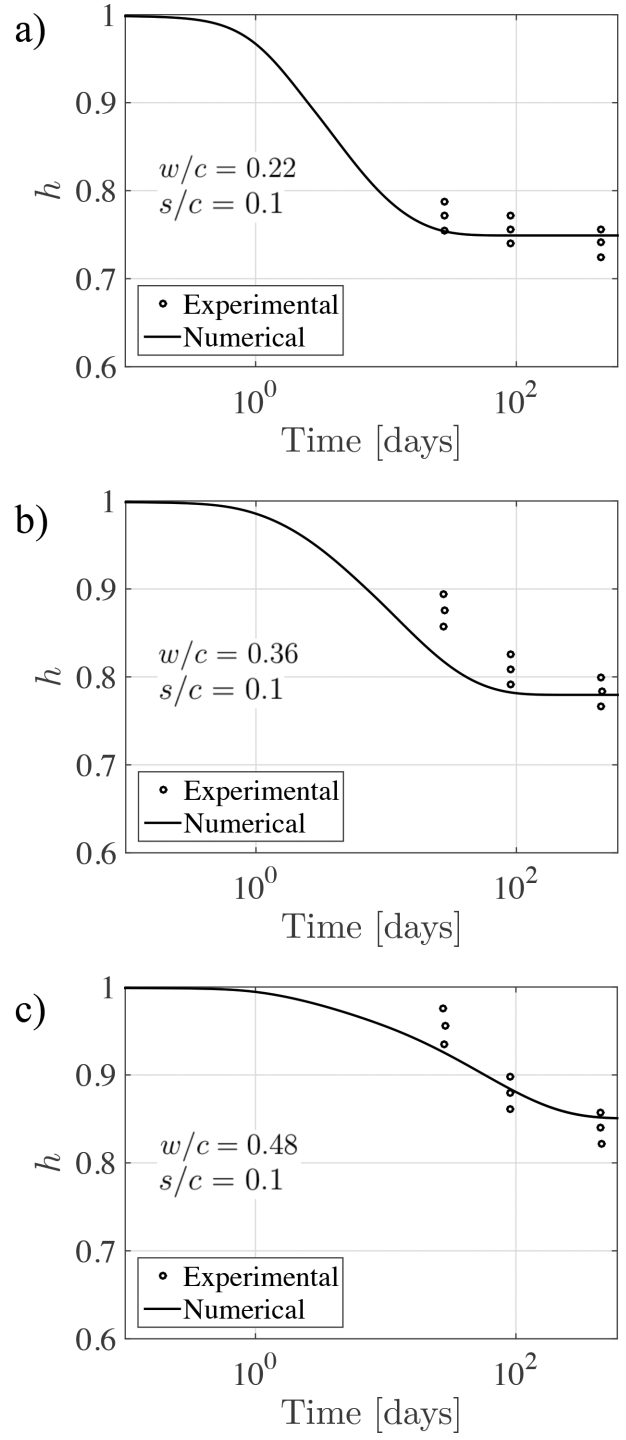

Figure 1. Prediction of self-desiccation in three different concretes. Upper and lower data points represent respectively $+/-5 \%$ in humidity (absolute). Experimental data is from Persson, 1996, 1997, 1998.

concrete ages. At 28 days, the absolute differences between experiments and simulations are $1.77 \%$, $5.92 \%, 3.05 \%$ respectively for the mixes with $w / c=$ $0.22, w / c=0.36$, and $w / c=0.48$. The predictions improve as time goes. Indeed, for the mix with $w / c=0.22$, the absolute differences are $0.70 \%$ and $0.81 \%$ respectively at 90 and 450 days. Similarly for the mix with $w / c=0.36$, one can report differences of respectively $2.61 \%$ and $0.34 \%$, whereas for the mix with $w / c=0.48$, one gets $0.40 \%$ and $1.17 \%$ respectively at 90 and 450 days. 


\section{CONCLUSIONS}

A multiscale parameter-free framework to simulate hydration in concrete has been presented, including the modeling of the cement microstructure (CEMHYD3D model) and mathematical formulations modeling the macro-scale hygro-thermochemical properties of concrete, in particular self-desiccation. The model results were also compared to experimental data. This preliminary study showed the following:

- It is possible to simulate self-desiccation using model predictions from the micro-scale without additional parameters from the experiments.

- The model is capable of accurately predicting the drop in internal humidity in time at ages of 90 days and beyond.

- The model can take into account a wide range of water to cement ratios and the inclusion of silica fume.

\section{ACKNOWLEDGMENT}

The work of the first and last authors was sponsored by the U.S. Army Engineer Research and Development Center (ERDC) under Contract Number W912HZ-17-C-0027. Permission to publish was granted by the director of the ERDC Geotechnical and Structures Laboratory.

\section{REFERENCES}

Bentz, D. (2005). Cemhyd3d: A three-dimensional cement hydration and microstructure development modeling package. version 3.0.

Bentz, D.P. (1997). Three-dimensional computer simulation of portland cement hydration and microstructure development. Journal of the American Ceramic Society 80(1), 3-21.

Bentz, D.P. \& E.J. Garboczi (1990). Digitised simulation model for microstructural development. Ceram. Trans. 16, 211-226.

Bishnoi, S. \& K.L. Scrivener (2009). $\mu$ ic: A new platform for modelling the hydration of cements. Cement and Concrete Research 39(4), 266-274.

Bullard, J.W. (2007a). Approximate rate constants for nonideal diffusion and their application in a stochastic model. The Journal of Physical Chemistry A 111(11), 2084-2092.

Bullard, J.W. (2007b). A three-dimensional microstructural model of reactions and transport in aqueous mineral systems. Modelling and Simulation in Materials Science and Engineering 15(7), 711.

Cervera, M., J. Oliver, \& T. Prato (1999). Thermochemomechanical model for concrete. i: Hydration and aging. Journal of engineering mechanics 125(9), 1018-1027.

De Schutter, G. \& L. Taerwe (1996). Degree of hydrationbased description of mechanical properties of early age concrete. Materials and Structures 29(6), 335.
Di Luzio, G. \& G. Cusatis (2009). Hygro-thermo-chemical modeling of high performance concrete. i: Theory. Cement and Concrete composites 31(5), 301-308.

Di Luzio, G. \& G. Cusatis (2013). Solidificationmicroprestress-microplane ( $\mathrm{smm}$ ) theory for concrete at early age: Theory, validation and application. International Journal of Solids and Structures 50(6), 957-975.

Garboczi, E. \& D. Bentz (1992). Computer simulation of the diffusivity of cement-based materials. Journal of materials science 27(8), 2083-2092.

Gawin, D., F. Pesavento, \& B.A. Schrefler (2006a). Hygrothermo-chemo-mechanical modelling of concrete at early ages and beyond. part i: hydration and hygro-thermal phenomena. International Journal for Numerical Methods in Engineering 67(3), 299-331.

Gawin, D., F. Pesavento, \& B.A. Schrefler (2006b). Hygrothermo-chemo-mechanical modelling of concrete at early ages and beyond. part ii: shrinkage and creep of concrete. International Journal for Numerical Methods in Engineering 67(3), 332-363.

Jennings, H.M. \& S.K. Johnson (1986). Simulation of microstructure development during the hydration of a cement compound. Journal of the American Ceramic Society 69(11), 790-795.

Koenders, E. \& K. Van Breugel (1997). Numerical modelling of autogenous shrinkage of hardening cement paste. Cement and Concrete Research 27(10), 1489-1499.

Molina, L. (1992). On predicting the influence of curing conditions on the degree of hydration. Cement och Betong Institututet.

Navi, P. \& C. Pignat (1996). Simulation of cement hydration and the connectivity of the capillary pore space. Advanced Cement Based Materials 4(2), 58-67.

Pan, Y. et al. (2017). Lattice modeling of early-age behavior of structural concrete. Materials 10(3), 231.

Persson, B. (1996). Hydration and strength of high performance concrete. Advanced Cement Based Materials 3(3), 107-123.

Persson, B. (1997, Nov). Moisture in concrete subjected to different kinds of curing. Materials and Structures 30(9), 533-544.

Persson, B. (1998). Seven-year study on the effect of silica fume in concrete. Advanced cement based materials 7(3), 139-155.

Rahimi-Aghdam, S., Z.P. Bažant \& M.A. Qomi (2017). Cement hydration from hours to centuries controlled by diffusion through barrier shells of csh. Journal of the Mechanics and Physics of Solids 99, 211-224.

Thomas, J.J. et al. (2011). Modeling and simulation of cement hydration kinetics and microstructure development. Cement and Concrete Research 41(12), 1257-1278.

Ulm, F.-J. \& O. Coussy (1995). Modeling of thermochemomechanical couplings of concrete at early ages. Journal of engineering mechanics 121(7), 785-794.

Van Breugel, K. (1995). Numerical simulation of hydration and microstructural development in hardening cement-based materials (i) theory. Cement and Concrete Research 25(2), 319-331.

Ye, G., K. Van Breugel, \& A. Fraaij (2003). Threedimensional microstructure analysis of numerically simulated cementitious materials. Cement and Concrete Research 33(2), 215-222. 